\title{
The GlueX Detector
}

\author{
D. Lawrence for the GlueX Collaboration
}

Thomas Jefferson National Accelerator Facility ${ }^{1}$

\begin{abstract}
The GlueX detector is being built along with the new experimental Hall D at Jefferson lab as part of the $12 \mathrm{GeV}$ upgrade project which received CD-3 approval in fall 2008. GlueX is a fixed target experiment built around a 2 Tesla superconducting solenoid having charged particle tracking and calorimetry with large acceptance. A high rate DAQ system consisting of pipeline electronics will allow the detector to operate at high luminosity ( $10^{8}$ tagged $\gamma / \mathrm{sec}$ on target). Details on the photon beam and GlueX detector are given including capabilities in resolutions and rates.
\end{abstract}

Keywords: GlueX, exotics, hybrids, flux tube

PACS: 12.38.Aw 12.38.Qk 12.39.Mk 13.60.Le

\section{INTRODUCTION}

The upgrade to $12 \mathrm{GeV}$ of the continuous wave electron accelerator at Jefferson Lab will provide high luminosity, high quality beams opening unique opportunities in the study of the quark and gluon structure of hadrons [1]. Construction of the new experimental Hall D and the GlueX detector will be done in support of a program to search for gluonic excitations using linearly polarized, real photons [2][3]. The photon beam will be generated through coherent bremstrahlung and tagged by a high luminosity, high resolution spectrometer. The GlueX detector is based on a superconducting solenoidal magnet. Drift chambers will be housed in the high field region of the magnet along with two complementary calorimeters (one inside and one outside the high field region) providing coverage for both charged and neutral particles over a wide angular range. A high rate DAQ system consisting of pipelined electronics will allow the detector to operate at high luminosity. Details on the photon beam and GlueX detector are given including capabilities in resolutions and rates.

\section{LINEARLY POLARIZED PHOTON BEAM}

The GlueX experiment will use a beam of tagged, linearly polarized photons. The photon beam is generated through coherent bremstrahlung from the primary electron beam provided by the accelerator by passing it through a $20 \mu \mathrm{m}$ diamond radiator. The crystalline structure of the diamond produces linearly polarized photons with enhancements in the energy spectrum that can be tuned by adjusting the angle of the diamond with respect

\footnotetext{
${ }^{1}$ Notice: Authored by Jefferson Science Associates, LLC under U.S. DOE Contract No. DE-AC0506OR23177. The U.S. Government retains a non-exclusive, paid-up, irrevocable, world-wide license to publish or reproduce this manuscript for U.S. Government purposes.
} 
to the electron beam. For the GlueX experiment, the peak will be tuned to $E_{\gamma}=9 \mathrm{GeV}$ which results in a polarization of approximately $40 \%$ after collimation.

The photons in the beam are "tagged" by measuring the residual energy of the postbremstrahlung electron via a $1.5 \mathrm{~T}$ dipole magnet. The electron is detected using two different arrays of scintillator detectors, one with wider energy coverage, and the other finer with better energy resolution, but covering a smaller energy range. The "Fixed Array" hodoscope consists of 192 scintillators covering the 3.0-11.7 GeV range with each counter spanning a $\sim 30 \mathrm{MeV}$ energy bite. In the energy range below $9 \mathrm{GeV}$, gaps between detectors exist such that there is only $50 \%$ coverage. Above $9 \mathrm{GeV}$, there is $100 \%$ coverage. The microscope array is made of $2 \mathrm{~mm} \times 2 \mathrm{~mm} \times 20 \mathrm{~mm}$ fibers which form a $100 \times 5$ grid as seen by the beam with the long dimension oriented along the bending plane of the electron beam. The fibers span $\sim 8 \mathrm{MeV}$ energy bites leading to about an $800 \mathrm{MeV}$ range in energy coverage. The microscope tags photons in the 8.39.1 GeV range for the GlueX experiment, it is designed to be relocatable in order to modify the energy range for additional measurements.

\section{GLUEX DETECTOR}

The GlueX Detector is a spectrometer built around $2 \mathrm{~T}$ superconducting solenoidal magnet. The GlueX experiment will be a fixed target experiment having linearly polarized photons incident on a $30 \mathrm{~cm}$ long $\mathrm{LH}_{2}$ target. The detector consists of two tracking chamber systems housed inside the solenoid and two EM calorimeters, one inside the solenoid and one downstream in the forward direction. Figure 1 shows a schematic cross-section of the detector. The following sections describe the major subsystems of the detector.

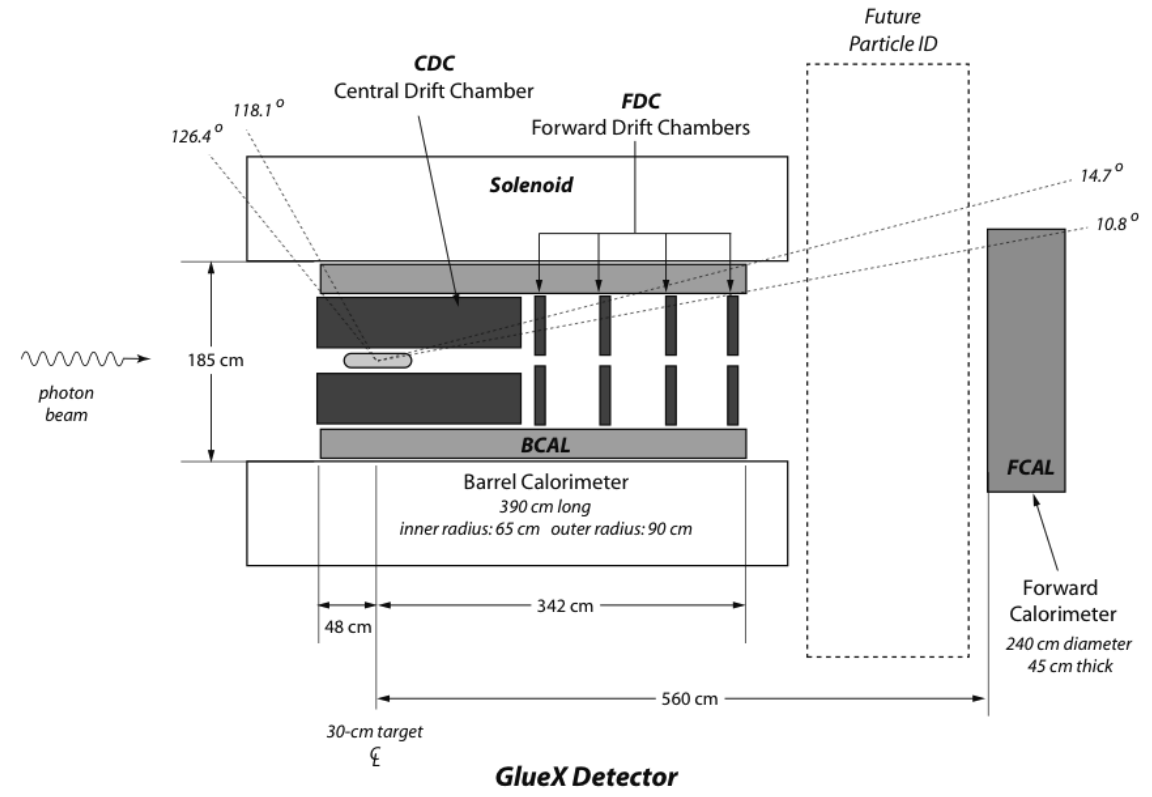

FIGURE 1. Diagram showing approximate locations of the major detector components of the GlueX detector. 


\section{Calorimetry}

The GlueX detector contains two EM calorimeter systems: The Barrel CALorimeter (BCAL) and the Forward CALorimeter (FCAL).

The BCAL is a sampling calorimeter made of a multi-layer lead/scintillating fiber sandwich with a sampling fraction of $12 \%$ (of energy). The system consists of 48 trapezoidal modules arranged in a barrel shape that lines the inside of the solenoid. Each module has a segmented readout consisting of 28 segments with finer segmentation on the inner part and coarser segmentation on the outer part. The BCAL segments are read out on both ends to give better noise suppression, position resolution, and timing resolution. In addition to calorimetry, the BCAL will give time-of-flight information for charged tracks (see section on particle identification). Beam tests with a prototype BCAL module [4] indicate a relative energy resolution of $\sigma_{E} / E=(5.54 / \sqrt{E} \oplus 1.6) \%$ and position resolution along the module of $\sigma_{z}=5.0 \mathrm{~mm} / \sqrt{E}$ which are consistent with results from KLOE for a similar calorimeter [5].

The FCAL consists of an array of 2800 lead-glass detectors. Individual blocks are $4 \mathrm{~cm} \times 4 \mathrm{~cm} \times 45 \mathrm{~cm}$. The majority of type F8-00 and are being recycled from the E852 experiment at Brookhaven National Lab [6]. The detectors near the beamline will be radiation hard F108 type lead-glass. The FCAL acceptance includes particles with $2^{\circ}<\theta<11^{\circ}$ and full azimuthal coverage. Experience with the E852 experiment indicates a relative energy resolution of $\sigma_{E} / E=(5.7 / \sqrt{E} \oplus 2.0) \%$ and position resolution of $\sigma_{x y}=6.4 \mathrm{~mm} / \sqrt{E}$. The PMTs will be powered by Cockcroft-Walton bases that generate high voltage internally requiring only a $12 \mathrm{~V}$ supply.

\section{Charged Particle Tracking}

The GlueX detector contains two charged particle tracking systems: the Central Drift Chambers (CDC) and the Forward Drift Chambers (FDC).

The CDC is a straw tube chamber consisting of 3522 straw tubes arranged in 28 layers radially from $\mathrm{R}=10.73 \mathrm{~cm}$ to $\mathrm{R}=54.76 \mathrm{~cm}$. The tubes are $150 \mathrm{~cm}$ long and alternate between sections of axial layers that are parallel to the beamline and stereo layers that are at an angle of about $6^{\circ}$ with respect to the beam direction. In all, there are twelve axial layers in three groups of four and sixteen stereo layers in four groups of four. The groups are arranged in order of increasing radius as AX, $-\mathrm{ST},+\mathrm{ST}, \mathrm{AX},+\mathrm{ST},-\mathrm{ST}, \mathrm{AX}$. Prototype studies with cosmic rays resulted in a position resolution of $\sigma_{r}=150 \mu \mathrm{m}$.

The FDC is a set of open-cell planar drift chambers with additional cathode strip readout. The FDC consists of 4 packages, each of which contains 6 tightly-packed chambers. Each chamber consists of 96 wires and 2 cathode planes, each having 216 copper strips. The cathode strips are at an angle with respect to the sense wires and therefore provide information on the location of the avalanche along the wire.The primary benefit provided by the cathode readout is improved pattern recognition since the avalanche locations provide 3D space points near the track. FDC prototype studies using cosmic rays have demonstrated a position resolution of $\sigma_{r}=200 \mu \mathrm{m}$ perpendicular to the wire from the drift time and $\sigma_{l}=200 \mu \mathrm{m}$ along the wire from the cathode strip information. 


\section{Particle Identification}

Particle identification in GlueX will come primarily from time-of-flight information with some additional capability for low energy particles using $d E / d x$ for energy deposited in the central drift chamber gas. For forward going particles $\left(2^{\circ}<\theta<11^{\circ}\right)$, the Time-Of-Flight (TOF) wall will provide timing information for charged tracks with $\sigma_{t o f}=60 \mathrm{ps}$ resolution [7]. The TOF consists of two planes each with fortyeight 1 inch thick scintillator paddles with two double-ended readout. For larger angle tracks $\left(12^{\circ}<\theta<140^{\circ}\right)$ the BCAL will provide timing information. Minimum ionizing hadrons passing through the BCAL will deposit $>200 \mathrm{MeV}$ and cosmic ray tests with a prototype module indicate $\sigma_{B C A L}=200 \mathrm{ps}$ timing resolution can be achieved.

The combination of the time-of-flight information and $d E / d x$ information should result in $\pi$ /proton separations for momenta up to $2.5 \mathrm{GeV} / \mathrm{c}$ in the forward direction $\left(\theta<11^{\circ}\right)$ and momenta up to $600 \mathrm{MeV} / \mathrm{c}$ in the wide-angle region $\left(\theta>12^{\circ}\right)$.

\section{Electronics and Data Rates}

The GlueX experiment will consist of digitization electronics which are fully pipelined with front-end memory capable of buffering over $3 \mu s$ of data which allows $3 \mu s$ to make and distribute a level 1 trigger decision. The pulse height information will be digitized using $125 \mathrm{MHz}$ and $250 \mathrm{MHz}$ flash ADC modules and the high resolution timing information will be digitized using a F1TDC module with 60 ps least count capability. All digitization electronics will be read out using the VME64x standard.

The integrated data rate from the front end modules will be $3 \mathrm{~GB} / \mathrm{s}$ at $10^{8} \mathrm{\gamma} / \mathrm{s}$. A level 3 trigger farm will be used to filter out about $90 \%$ of the events leaving a final data rate to tape of $300 \mathrm{MB} / \mathrm{s}$ resulting in about $3 \mathrm{~PB} / \mathrm{yr}$ going to mass storage.

\section{SCHEDULE}

At the time of this writing (Summer 2009) site preparation for Hall D is underway with civil construction of the experimental hall taking place over the next year. Beneficial occupancy of Hall-D is scheduled to begin August 2010 with installation of the magnet into the tagger hall about one year later in November 2011. Commissioning of the detector is planned to begin in April 2014.

\section{SUMMARY}

The GlueX experiment will attempt to map out the spectrum of light-quark, hybrid mesons, including ones with exotic quantum numbers, using a new high-acceptance, high-resolution spectrometer being built along with a new experimental hall (Hall D) at Jefferson Lab. GlueX will be a fixed target experiment using a linearly polarized photon beam $\left(E_{\gamma} \approx 9 \mathrm{GeV}\right)$ incident on a $30 \mathrm{~cm} \mathrm{LH}_{2}$ target. Table 1 summarizes the GlueX detector capabilities. 
TABLE 1. GlueX Detector Design Parameters

\begin{tabular}{lll}
\hline Detector/System & Property & Resolution / Range \\
\hline Photon Beam & Linear polarization & $\sim 40 \%$ (varies with energy) \\
& Energy range(microscope) & $8.3-9.1 \mathrm{GeV}$ (adjustable) \\
& Energy resolution (microscope) & $\sim 8 \mathrm{MeV}$ \\
& Energy range(fixed array) & $3.0-11.7 \mathrm{GeV}$ \\
& Energy resolution (fixed array) & $\sim 30 \mathrm{MeV}$ \\
\hline Charged Particles & Angular coverage & $1^{\circ}<\theta<155^{\circ}$ \\
& Momentum resolution $\left(5^{\circ}-140^{\circ}\right)$ & $\sigma_{p} / p=1-3 \%$ \\
& Position resolution & $\sigma_{x}=150-200 \mu m$ \\
& dE/dx (chamber gas) & $20^{\circ}<\theta<155^{\circ}$ \\
& Time-of-flight $\left(1^{\circ}-11^{\circ}\right)$ & $\sigma_{t}=60 \mathrm{ps}$ \\
& Time-of-flight $\left(12^{\circ}-140^{\circ}\right)$ & $\sigma_{t}=200 \mathrm{ps}$ \\
\hline Photon Detection & Angular coverage & $2^{\circ}<\theta<120^{\circ}$ \\
& Energy resolution $\left(1^{\circ}-11^{\circ}\right)$ & $\sigma_{E} / E=(5.7 / \sqrt{E} \oplus 2.0 \%)$ \\
& Energy resolution $\left(12^{\circ}-140^{\circ}\right)$ & $\sigma_{E} / E=(5.54 / \sqrt{E} \oplus 1.6 \%)$ \\
& Position resolution (forward) & $\sigma_{x, y}=0.64 \mathrm{~cm} / \sqrt{E}$ \\
& Position resolution (barrel) & $\sigma_{z}=0.5 \mathrm{~cm} / \sqrt{E}$ \\
\hline DAQ/trigger & Level 1 & $200 \mathrm{kHz}$ \\
& L1 Trigger latency & $3.2 \mu s$ \\
& Event rate to tape & $15 \mathrm{kHz}$ \\
& Data rate to tape & $300 \mathrm{MB} / \mathrm{s}$ \\
\hline Digitization Electronics & Fully pipelined & $125 / 250 \mathrm{MHz} \mathrm{fADCs}, \mathrm{TDCs}$ \\
\hline Photon Flux & Low luminosity (commissioning) & $10^{7} \gamma / \mathrm{s}$ \\
& High luminosity & $10^{8} \gamma / \mathrm{s}$ \\
\hline & &
\end{tabular}

\section{ACKNOWLEDGMENTS}

\section{REFERENCES}

1. E. Smith, arXiv:0901.3249/nucl-ex (2008).

2. M. Shepherd, CIPANP2009 (these proceedings) (2009).

3. A. R. Dzierba, hep-ex/0106010 (2001).

4. B. D. Leverington, et al., Nucl. Instrum. and Meth. A 596, 327-337 (2008).

5. M. Adinolfi, et al., Nucl. Instrum. and Meth. A 494, 326-331 (2002).

6. R. R. Crittenden, et al., Nucl. Instrum. and Meth. A 387, 377-394 (1997).

7. S. Denisov, et al., Nucl. Instrum. and Meth. A 525, 183-187 (2004). 